\title{
Molecular and functional characterization of the adiponectin (AdipoQ) gene in goat skeletal muscle satellite cells
}

\author{
Linjie Wang ${ }^{1, a}$, Ke Xue ${ }^{1, a}$, Yan Wang ${ }^{1}$, Lili Niu ${ }^{1}$, Li Li ${ }^{1}$, Tao Zhong ${ }^{1}$, Jiazhong Guo ${ }^{1}$, Jing Feng ${ }^{2}$, \\ Tianzeng Song ${ }^{2}$, and Hongping Zhang ${ }^{1, *}$
}

\author{
* Corresponding Author: Hongping Zhang \\ Tel: +86-28-86291010, Fax: +86-28-86290987 \\ E-mail: zhp@sicau.edu.cn \\ ${ }^{1}$ Institute of Animal Genetics and Breeding, College of \\ Animal Science and Technology, Sichuan Agricultural \\ University, Chengdu, Sichuan, China \\ ${ }^{2}$ Institute of Animal Science, Tibet Academy of \\ Agricultural \& Animal Husbandry Science, Lhasa, \\ China \\ a These authors contributed equally to this work. \\ ORCID \\ Hongping Zhang \\ https://orcid.org/0000-0001-8290-7041
}

Submitted May 27, 2017; Revised Sept 1, 2017; Accepted Jan 30, 2018
Objective: It is commonly accepted that adiponectin binds to its two receptors to regulate fatty acid metabolism in adipocytes. To better understand their functions in the regulation of intramuscular adipogenesis in goats, we cloned the three genes (adiponectin [AdipoQ], adiponectin receptor 1 [AdipoR1], and AdipoR2) encoding these proteins and detected their $\mathrm{mRNA}$ distribution in different tissues. We also determined the role of AdipoQ in the adipogenic differentiation of goat skeletal muscle satellite cells (SMSCs).

Methods: SMSCs were isolated using $1 \mathrm{mg} / \mathrm{mL}$ Pronase $\mathrm{E}$ from the longissimus dorsi muscles of 3-day-old female Nanjiang brown goats. Adipogenic differentiation was induced in satellite cells by transferring the cells to Dulbecco's modified Eagle's medium supplemented with an isobutylmethylxanthine, dexamethasone and insulin cocktail. The pEGFP-N1-AD plasmid was transfected into SMSCs using Lipofectamine 2000. Expression of adiponectin in tissues and SMSCs was detected by quantitative polymerase chain reaction and immunocytochemical staining.

Results: The three genes were predominantly expressed in adipose and skeletal muscle tissues. According to fluorescence and immunocytochemical analyses, adiponectin protein expression was only observed in the cytoplasm, suggesting that adiponectin is localized to the cytoplasm of goat SMSCs. In SMSCs overexpressing the AdipoQ gene, adiponectin promoted SMSC differentiation into adipocytes and significantly $(\mathrm{p}<0.05)$ up-regulated expression of AdipoR2, acetyl-CoA carboxylase, fatty-acid synthase, and sterol regulatory element-binding protein-1, though expression of CCAAT/enhancer-binding protein- $\alpha$, peroxisome proliferatoractivated receptor $\gamma$, and AdipoR1 did not change significantly.

Conclusion: Adiponectin induced SMSC differentiation into adipocytes, indicating that adiponectin may promote intramuscular adipogenesis in goat SMSC.

Keywords: Adiponectin; Intramuscular Adipogenesis; Skeletal Muscle Satellite Cells; Fatty Acid Synthesis

\section{INTRODUCTION}

Adiponectin, an adipokine belonging to the complement factor C1Q protein family [1], is expressed in liver sinusoid endothelial cells, skeletal muscle and bone-forming cells as well as in adipocytes [2-4]. Adiponectin, which is expressed by the AdipoQ gene, consists of four parts: an amino-terminal signal peptide, a variable region, a collagenous domain and a carboxyl-terminal globular domain [5]. There are two receptors for adiponectin, AdipoR1 and AdipoR2. Both are seven-transmembrane domain proteins with an intracellular N-terminus and highly expressed in skeletal muscle (AdipoR1) and adipose tissue (AdipoR2) [6]. Adiponectin circulates in the plasma in several forms: globular adiponectin (gAd) interacts with AdipoR1; whereas full-length adiponectin (fAd) binds to both AdipoR1 and AdipoR2 
to regulate glucose and fatty acid metabolism [7].

Emerging evidence suggests that adiponectin enhances glucose uptake by increasing GLUT4 translocation to the cell membrane [8] and promotes fatty acid oxidation by activating adenosine 5'-monophosphate (AMP)-activated protein kinase (AMPK), p38-mitogen-activated protein kinase, and peroxisome proliferator-activated receptor alpha pathways $[9,10]$. In addition, adiponectin inhibits gluconeogenesis by phosphorylating AMPK and acetyl-CoA carboxylase (ACC) [11]. For example, intravenous infusion of adiponectin in mice leads to lower expression of PEPCK and G6Pase in the liver, which inhibits endogenous glucose production [12]. In myocytes, fAd increases the number of mitochondria and enhances fatty acid oxidation by activating CaMKK $\beta$ and PGC1- $\alpha$ [13]. Studies have also reported that overexpression of the Adipo $Q$ gene in fat tissue and fibroblasts results in increased lipid accumulation [14]. Therefore, the results of these studies suggest that adiponectin plays important roles in regulating glucose uptake and fatty acid metabolism.

It has been shown that ACC and fatty-acid synthase (FASN) are two key enzymes in de novo fatty acid synthesis. ACC couples with FASN to increase fatty acid production in Escherichia coli and humans $[15,16]$. In addition, sterol regulatory elementbinding protein-1 (SREBP-1), CCAAT/enhancer-binding protein- $\alpha(\mathrm{C} / \mathrm{EBP} \alpha)$ and peroxisome proliferator-activated receptor $\gamma(\operatorname{PPAR} \gamma)$ are master adipogenic transcription factors that have been proven to promote the formation of white adipose tissue [17]. As previously demonstrated, PPAR $\gamma$ and SREBP-1 cooperatively play key roles in regulating the redifferentiation of bovine adipofibroblasts [18]. Moreover, C/EBP $\alpha$ has important functions in regulating cell proliferation and differentiation, especially adipocyte differentiation [19], and PPAR $\gamma$ and C/EBPa together induce adipogenesis and adipocyte differentiation [20]. These studies demonstrate that these genes play pivotal roles in fatty acid synthesis and adipocyte differentiation. Although adiponectin has been shown to regulate fatty acid metabolism, it remains unknown whether adiponectin regulates the expression levels of these adipogenic-related genes.

An increasing number of studies on adiponectin are being carried out in humans and mice, but information on the gene encoding adiponectin in domestic animals is still limited. In addition, the role of adiponectin in the adipogenic differentiation of muscle satellite cells is unknown. In this study, we cloned and characterized the goat AdipoQ, AdipoR1, and AdipoR2 genes and detected their mRNA expression levels in different tissues. In addition, we quantified the expression levels of these adipogenic-related genes in skeletal muscle satellite cells (SMSCs) overexpressing adiponectin. The results of this study provide information on the expression patterns of the AdipoQ, AdipoR1, and AdipoR2 genes in goat as well as the functions of adiponectin in regulating fatty acid synthesis.

\section{MATERIALS AND METHODS}

\begin{abstract}
Animals and tissue collection
All procedures were performed according to the guidelines established by the Sichuan Province Committee for the Protection of Laboratory Animals. Eleven tissues, namely, the ovary, spleen, rumen, liver, brain, heart, gastrocnemius muscle, biceps femoris muscle, longissimus dorsi muscle (LD), and mesenteric and subcutaneous adipose tissue, were dissected from three three-day-old female Nanjiang brown goats. All tissues were immediately frozen in liquid nitrogen and then stored at $-80^{\circ} \mathrm{C}$.
\end{abstract}

\section{RNA isolation and cDNA synthesis}

Total RNA was extracted by using TRIzol reagent (Invitrogen, Carlsbad, CA, USA) according to the manufacturer's protocol. The integrity of total RNA was examined by agarose gel electrophoresis, and the purity and concentration were determined using a NanoDrop (Thermo Fisher, Waltham, MA, USA) instrument. First-strand complementary DNA (cDNA) was synthesized using $2 \mu \mathrm{g}$ of total RNA and a PrimeScript RT Reagent Kit (TaKaRa, Tokyo, Japan) according to the manufacturer's protocol.

\section{Primer design and gene cloning}

Based on the conserved regions of the human and cattle $A d$ $i p o Q$, AdipoR1, and AdipoR2 gene sequences, primer pairs for gene cloning and quantitative polymerase chain reaction (qPCR) were designed by Primer Premier 5.0 (Table 1) and synthesized by Shanghai Sangon Biotechnology Company (Shanghai, China). The total reaction volume was $10 \mu \mathrm{L}$ and included $1 \mu \mathrm{L}$ cDNA, $5 \mu \mathrm{L} 2 \times$ Taq PCR Master Mix, $0.8 \mu \mathrm{L}$ $(8 \mathrm{pM})$ primer pairs (ADF/R for AdipoQ, ADR1F/R for Adi-

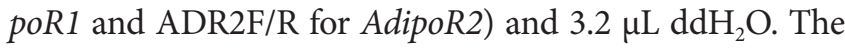
PCR procedures were as follows: initial denaturation at $95^{\circ} \mathrm{C}$ for $3 \mathrm{~min} ; 35$ cycles of $95^{\circ} \mathrm{C}$ for $30 \mathrm{~s}$, optimum annealing temperature (Table 1) for $30 \mathrm{~s}$ and $72^{\circ} \mathrm{C}$ for 1 to $2 \mathrm{~min}$; and a final extension at $72^{\circ} \mathrm{C}$ for $5 \mathrm{~min}$. All products were separated by agarose gel electrophoresis, purified using a gel extraction reagent kit (Tiangen, Beijing, China), cloned into the pMD19-T vector (TaKaRa, Japan) and sequenced by Shanghai Invitrogen Biotechnology Company (Shanghai, China).

\section{Semi-quantitative reverse transcription-PCR analysis}

To detect the mRNA tissue distributions of the AdipoQ, AdipoR1, and AdipoR2 genes, we performed semi-quantitative reverse transcription (RT)-PCR. The total reaction volume was $20 \mu \mathrm{L}$ and included $2 \mu \mathrm{L}$ cDNA, $10 \mu \mathrm{L} 2 \times$ Taq PCR Master Mix, $1.2 \mu \mathrm{L}(12 \mathrm{pM})$ primer pairs (ADQF/R for AdipoQ, ADR1QF/R for AdipoR1 and ADR2QF/R for AdipoR2) and

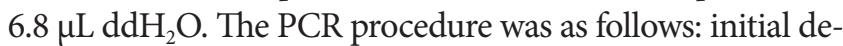
naturation at $95^{\circ} \mathrm{C}$ for $3 \mathrm{~min} ; 26$ to 32 cycles at $95^{\circ} \mathrm{C}$ for $30 \mathrm{~s}$, 
Table 1. Primer pairs used in this study

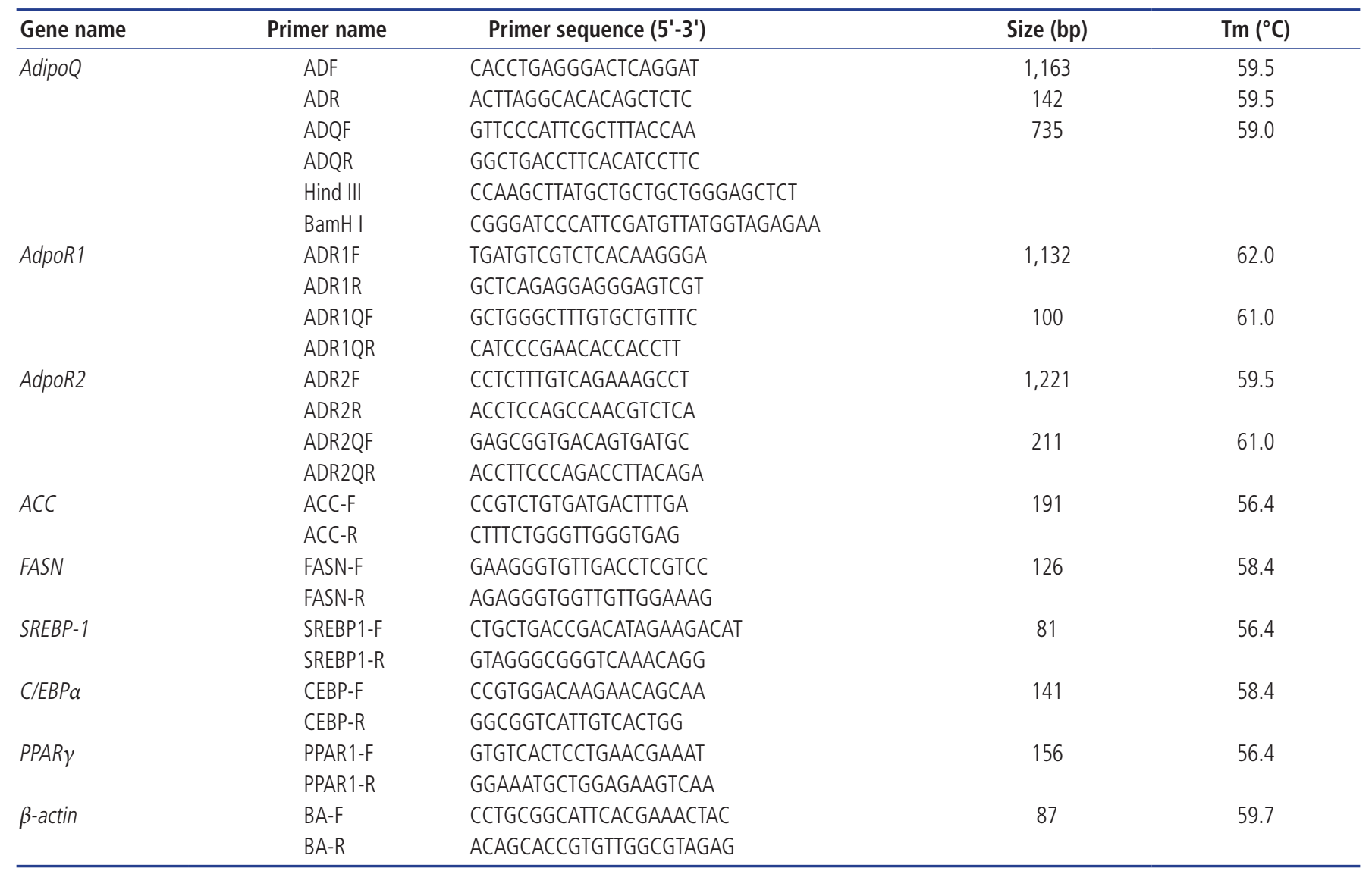

AdipoQ, adiponectin; AdpoR, adiponectin receptor; ACC, acetyl-CoA carboxylase; FASN, fatty-acid synthase; SREBP-1, sterol regulatory element-binding protein-1; C/EBPa, CCAAT/enhancer-binding protein- $\alpha$; PPAR $\gamma$, peroxisome proliferator-activated receptor $\gamma$.

optimum annealing temperature (Table 1) for $30 \mathrm{~s}$ and $72^{\circ} \mathrm{C}$ for $10 \mathrm{~s}$; and a final extension at $72^{\circ} \mathrm{C}$ for $5 \mathrm{~min}$. All products were examined by agarose gel electrophoresis to estimate mRNA abundance in the 11 tissues tested; $\beta$-actin was used as an internal control gene.

\section{Quantitative real-time PCR}

qPCR was carried out using a SYBR Green-based kit in $10-\mu \mathrm{L}$ reaction volumes containing $5 \mu \mathrm{L}$ SYBR Green real-time PCR Master Mix (TaKaRa, Japan), $0.8 \mu \mathrm{L}$ cDNA and $0.4 \mu \mathrm{L}$ each primer; a Bio-Rad CFX96 qPCR instrument (Bio-Rad, Hercules, CA, USA) was used. The qPCR procedure was as follows: initial denaturation at $95^{\circ} \mathrm{C}$ for $3 \mathrm{~min} ; 40$ cycles at $95^{\circ} \mathrm{C}$ for $30 \mathrm{~s}$, alternative annealing for $20 \mathrm{~s}$, and $72^{\circ} \mathrm{C}$ for 15 $\mathrm{s}$; and a final extension for $5 \mathrm{~min}$. Melting curve analysis was used to confirm specific PCR products. Gene expression levels were quantified relative to $\beta$-actin expression using the comparative $\mathrm{Ct}\left(2^{-\Delta \Delta \mathrm{Ct}}\right)$ value method.

\section{Cell culture}

SMSCs were isolated using $1 \mathrm{mg} / \mathrm{mL}$ Pronase E (Sigma, St. Louis, MO, USA) from the longissimus dorsi muscles from 3-day-old female Nanjiang brown goats. After enzymatic digestion, cells were separated from tissue fragments by repeated centrifugation at $500 \times \mathrm{g}$ for $5 \mathrm{~min}$, followed by filtration through a $200-\mu \mathrm{m}$ filter and then a 50- $\mu \mathrm{m}$ Nytex filter. SMSCs were cultured in Dulbecco's modified Eagle's medium (DMEM) containing $10 \%(\mathrm{v} / \mathrm{v})$ fetal bovine serum, $100 \mathrm{U} / \mathrm{mL}$ penicillin, and $0.1 \mathrm{mg} / \mathrm{mL}$ streptomycin under conditions of $5 \% \mathrm{CO}_{2}$ and $37^{\circ} \mathrm{C}$. When the cells had grown to $80 \%$ to $90 \%$ confluence, adipogenic differentiation of satellite cells was induced by transferring the cells into DMEM supplemented with an isobutylmethylxanthine, dexamethasone and insulin (MDI) cocktail (0.5 mM 1-methyl-3-isobutylxanthine, $1 \mu \mathrm{M}$ dexamethasone, and $10 \mu \mathrm{g} / \mathrm{mL}$ insulin), followed by supplementation with $10 \mu \mathrm{g} / \mathrm{mL}$ insulin for 1 day. The medium was replaced every two days. Cells were visualized by staining with oil red O solution.

\section{Plasmid construction and transient transfection} Using Primer Premier 5.0, a primer pair (Hind III-F, BamH $\mathrm{I}-\mathrm{R}$ ) was designed to amplify the coding region of Adipo $Q$ for plasmid construction. PCR products and the pEGFP-N1 (Clontech, San Francisco, CA, USA) vector were digested with 
Hind III and BamH I restriction enzymes (TaKaRa, Japan), purified with a gel extraction reagent kit and ligated using T4 DNA ligase (TaKaRa, Japan). The resulting plasmid (pEGFP-N1-AD) was sequenced to confirm the correct orientation and reading frame.

Transient transfection was performed according to the manufacturer's protocol: $4 \mu \mathrm{g}$ plasmid and $10 \mu \mathrm{L}$ Lipofectamine 2000 (Invitrogen, Carlsbad, CA, USA) were mixed, cultured for $20 \mathrm{~min}$ and then transfected into cells. Twenty-four hours after transfection, the cells were rinsed with phosphate-buffered saline; a portion was fixed with $4 \%$ formaldehyde for 30 $\mathrm{min}$ at room temperature to detect the cellular distribution of the adiponectin fusion protein using a fluorescence microscope (Nikon, Tokyo, Japan); other cells were suspended in TRIzol reagent for RNA extraction and expression analysis. The results were normalized to the expression of $\beta$-actin, and the expression of the control group (pEGFP-N1) was set as 1 for expression analysis of each gene. The relative mRNA expression analysis was performed using SAS 9.0 software with the generalized linear model process. Duncan's new multiple range test was applied to analyze statistical significance.

\section{Immunocytochemistry}

Expression of adiponectin in SMSCs was detected by immunocytochemical staining. Briefly, cells were fixed with $4 \%$ paraformaldehyde for $30 \mathrm{~min}$ and then permeabilized in $0.25 \%$ Triton X-100 with shaking at room temperature for $30 \mathrm{~min}$, followed by blocking with $1 \%$ bovine serum albumin for $1 \mathrm{~h}$. The cells were then incubated overnight at $4^{\circ} \mathrm{C}$ with an anti-adiponectin primary antibody (1:100) (Boster Company, Wuhan, China). The secondary antibody, horseradish peroxidase-conjugated goat anti-rabbit immunoglobulin G (1:200), was incubated with the cells for $30 \mathrm{~min}$. Cells incubated without the primary antibody were used as the negative control.

\section{Bioinformatic analysis}

ORF Finder (http://www.ncbi.nlm.nihgov/gorf/gorf.html) and DNASTAR were used to predict the open reading frames of the three genes. The domains of the three proteins were predicted by ProtParam (http://cn.expasy.org/tools/protparam. html) and SMART (http://smart.embl-heidelberg.de/), and the functional domain diagram was generated with DOG 2.0 software [21]. Amino acid sequences were aligned using ClustalX (http://www.ebi.ac.uk/clustalw/).

\section{RESULTS}

\section{Molecular characterization of AdipoQ, AdipoR1, and AdipoR2}

The full-length coding sequences of the goat AdipoQ, AdipoR1, and AdipoR2 genes were obtained (accession numbers:
JX573539, KC286912 and JX573540). The coding regions of the AdipoQ, AdipoR1, and AdipoR2 are 720 bp, 1,128 bp, and 1,176 bp, respectively. The AdipoQ gene encodes a 239-aa protein with a molecular weight of $25.95 \mathrm{kDa}$. The AdipoR1 gene encodes a 375-aa protein with a molecular weight of 42.41 $\mathrm{kDa}$, and the AdipoR2 gene encodes a 391-aa protein with a molecular weight of $43.81 \mathrm{kDa}$. These three genes share high similarity with other mammalian orthologous genes (Supplementary Table S1). Goat AdipoR1 shows the highest homology with other mammals, with the amino acid sequence sharing more than $96 \%$ identity with other mammalian sequences. Goat AdipoQ and AdipoR2 exhibit relatively high homology with other mammalian sequences. The amino acid sequence of AdipoQ is $81.17 \%, 87.45 \%, 81.59 \%$, and $99.16 \%$ identical to those of Homo sapiens, Bos taurus, Mus musculus, and Ovis aries, respectively. The amino acid sequence of AdipoR 2 shows similar identity to those of humans (86.27\%), Bos taurus (92.49\%), Mus musculus (84.20\%), and Ovis aries (94.04\%). Prediction of the deduced protein structure showed that adiponectin harbors a 137-aa C1Q domain; although the receptors both contain a 220 -aa seven-transmembrane domain, these receptors are not identical (Figure 1).

\section{Tissue distribution of goat AdipoQ, AdipoR1, and AdipoR2}

The mRNA tissue distribution of these three genes was detected by semi-quantitative RT-PCR, and the agarose gel electrophoresis results are shown in Figure 2. AdipoQ expression was predominantly observed in mesenteric and subcutaneous adipose tissues but was weakly expressed in the gastrocnemius muscle, LD muscle and biceps femoris muscle; expression was not detected in other tissues. AdipoR1 mRNA was found to be expressed in almost all tissues, with high expression in skeletal muscles and the rumen, followed by adipose tissue; its expression in other tissues was relatively weak. Similarly, AdipoR2 was expressed in all tissues but was most abundant in adipose and skeletal muscle tissues; it was weakly expressed in other tissues. The expression patterns of these three genes were further quantitated by qPCR (Figure 3). AdipoQ mRNA was predominantly expressed in adipose tissue, whereas its expression in skeletal muscles was relatively weak. AdipoR1 and AdipoR2 mRNA were expressed in all tissues examined, with the highest expression in adipose and skeletal muscle tissues.

\section{Subcellular localization of goat adiponectin in SMSCs}

Fluorescence analysis showed a green fluorescent signal throughout cells of the control group transfected with the pEGFP-N1 plasmid; In contrast, the green fluorescence of the adiponectin fusion protein was only observed in the cytoplasm of cells transfected with the pEGFP-AD plasmid (Figure 4), suggesting that adiponectin is localized to the cytoplasm. 


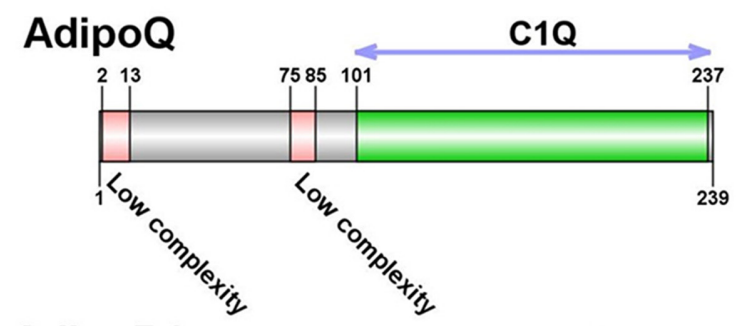

AdipoR1

transmembranes

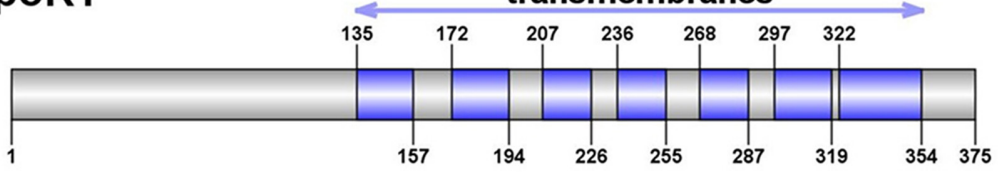

AdipoR2

transmembranes

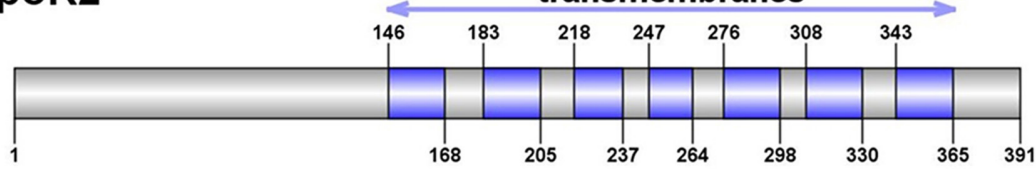

Figure 1. Diagram of the deduced protein structures of AdipoQ, AdipoR1, and AdipoR2. Adiponectin has a C1Q domain, and AdipoR1 and AdipoR2 both possess a seventransmembrane domain. Domains are color coded: pink indicates low-complexity segments, green indicates the C1Q domain, and blue indicates transmembrane domains. AdipoQ, adiponectin; AdpoR, adiponectin receptor.

Furthermore, immunostaining in SMSCs using an antibody against the human adiponectin protein revealed strong positive staining within the goat SMSC cytoplasm (Figure 5A) compared with the controls (Figure 5B).

\section{Adiponectin promotes fatty acid synthesis in skeletal} muscle satellite cells

To determine the role of adiponectin in the adipogenic differentiation of goat MSCs, cells were transfected with the pEGFP-N1-AD plasmid to overexpress the goat AdipoQ gene. To evaluate effective overexpression of adiponectin, whole-cell lysates were harvested for analysis of expression levels. In SMSCs, adiponectin was overexpressed approximately 80fold compared with that in control cells transfected with the
pEGFP-N1 vector (Figure 6). These results demonstrate that cells transfected with the pEGFP-N1-AD plasmid can overexpress adiponectin compared with the expression levels of control goat SMSCs expressing green fluorescent protein (GFP).

Next, satellite cells were induced with an MDI adipogenic cocktail and stained with oil red O seven days later. As shown in Figure 7, more oil red O-positive cells were observed in the pEGFP-N1-AD-transfected group than in the control group, indicating that adiponectin promoted MSC differentiate into adipocytes. In addition, several markers of adipogenic differentiation were detected by qPCR. The results showed that adiponectin overexpression significantly $(\mathrm{p}<0.01)$ up-regulated the mRNA expression levels of AdipoR2, ACC, FAS, and SREBP-1 (Figure 8), which were 1.85, 4.55, 4.88, and 4.25 times

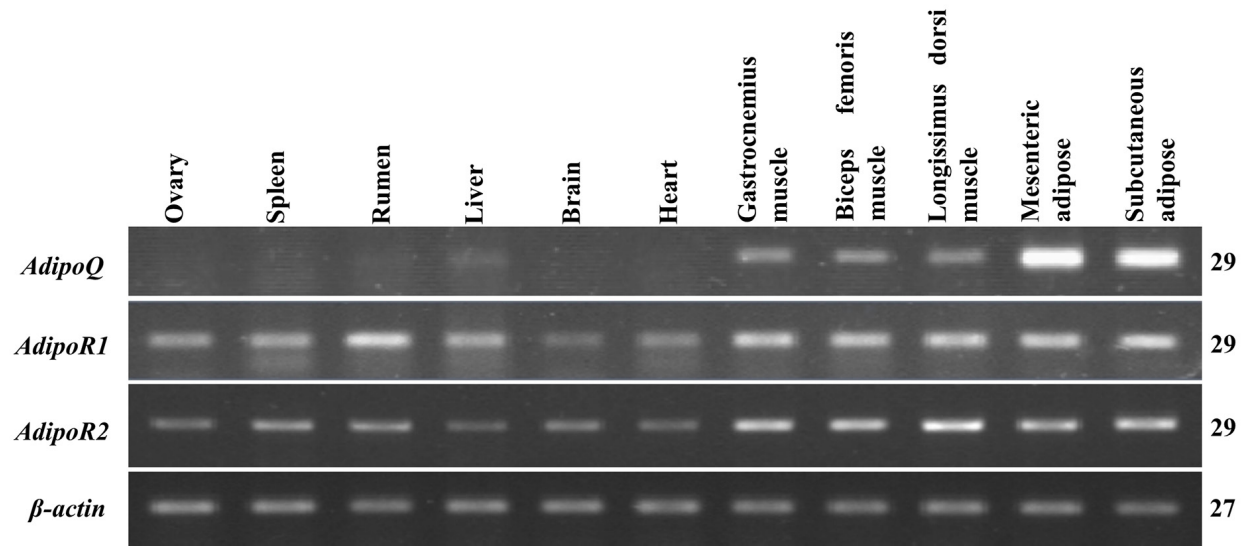

Figure 2. mRNA distribution of AdipoQ, AdipoR1, and AdipoR2 genes in 11 tissues of three-day-old female Nanjiang brown goats; $\beta$-actin was used as the internal standard control gene. Cycle numbers are indicated in the right margin. AdipoQ, adiponectin; AdpoR, adiponectin receptor. 

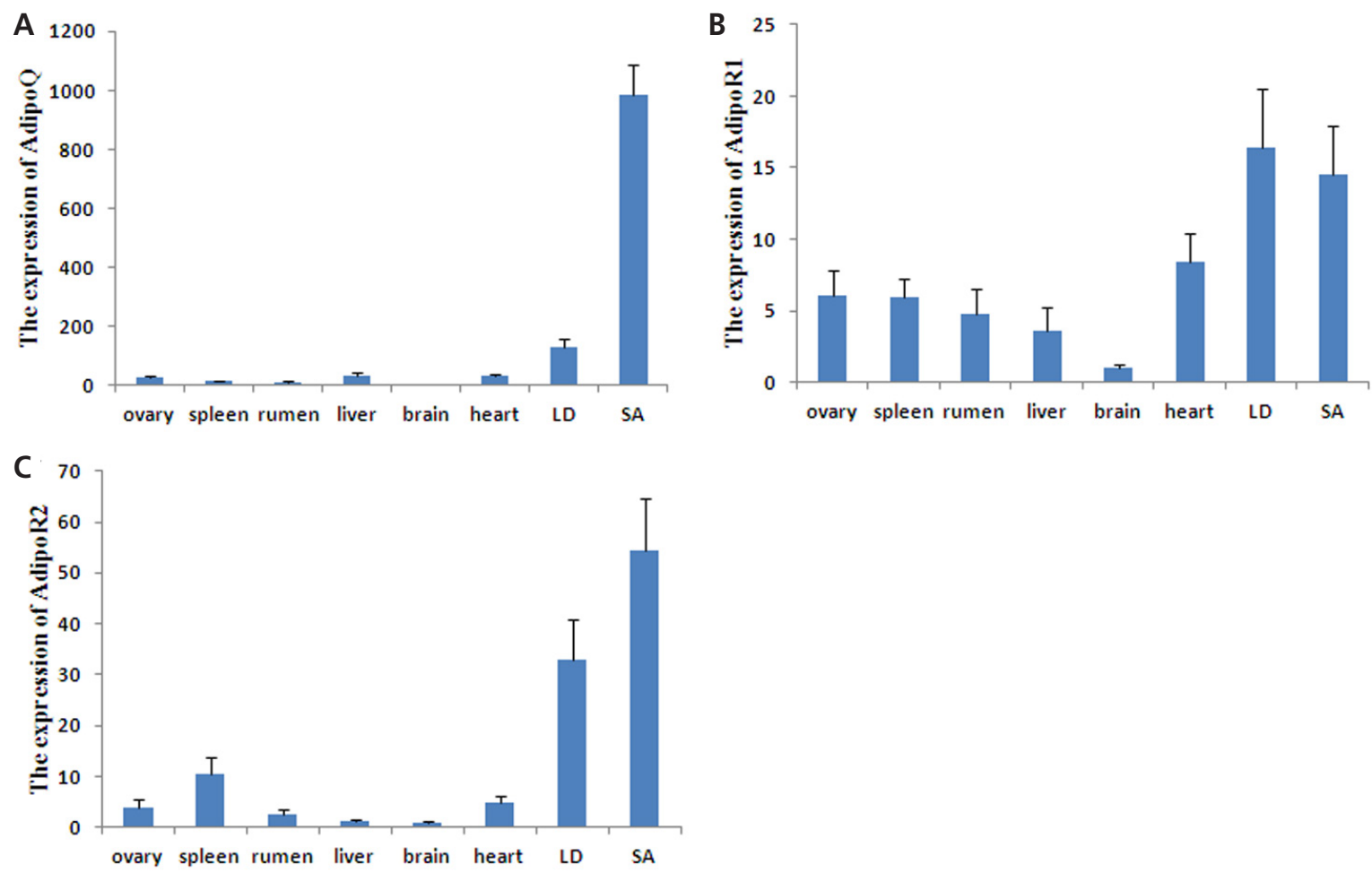

Figure 3. mRNA expression levels of AdipoQ, AdipoR1, and AdipoR2 in different tissues were determined by quantitative polymerase chain reaction. The samples represent the longissimus dorsi muscle (LD) and subcutaneous adipose (SA) tissue. The bars represent the mean \pm standard error of the mean. $(n=3) ; \beta$-actin was used as a control. AdipoQ, adiponectin; AdpoR, adiponectin receptor.

higher, respectively, than those of the control group. Conversely, no significant changes in expression were found for $C / E B P \alpha$, PPAR $\gamma$, and AdipoR1 (data not shown).

\section{DISCUSSION}

Recently, many studies have focused on the functions of the
AdipoQ gene in regulating glucose and fat metabolism and on its anti-inflammatory and anti-atherosclerosis effects in some species [12,22,23]. In the present study, we cloned the goat AdipoQ, AdipoR1, and AdipoR2 genes to investigate their expression and the functions of the encoded proteins. We found these gene sequences to be conserved among species. Adiponectin belongs to the complement factor C1Q family,

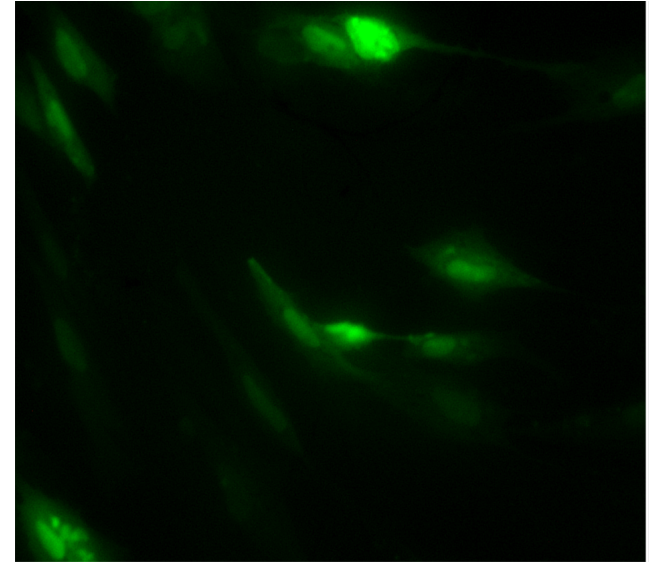

pEGFP-N1

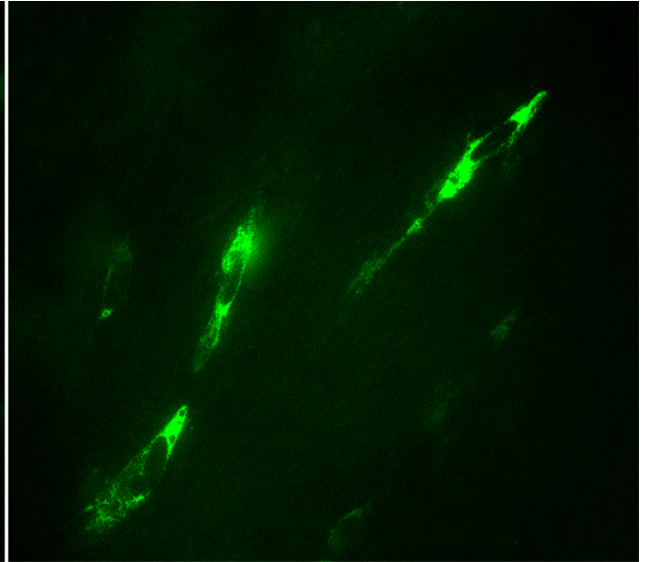

pEGFP-N1-AD

Figure 4. Adiponectin protein subcellular localization in skeletal muscle satellite cells (SMSCs). Green fluorescent protein (GFP) in the control group was expressed throughout the cell, whereas the GFP-adiponectin fusion protein was only found in the cytoplasm. 

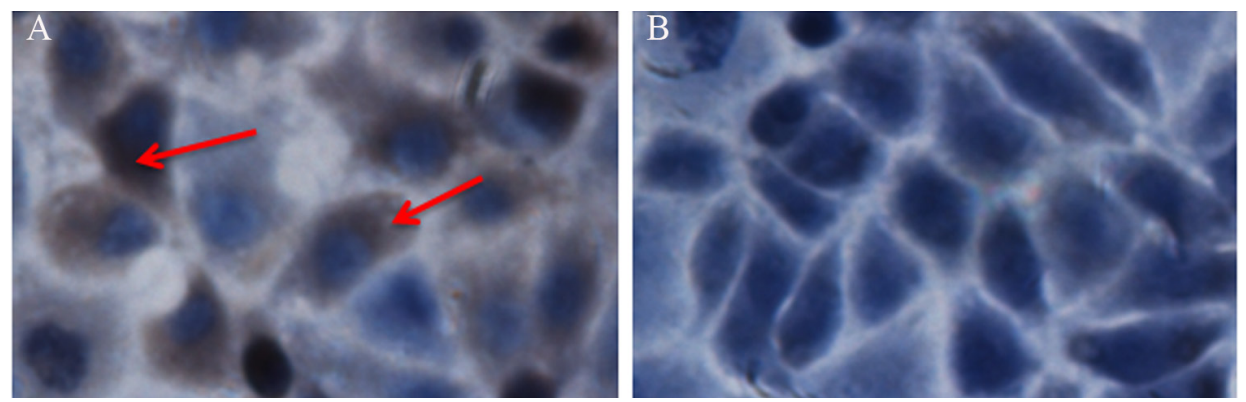

Figure 5. Adiponectin protein expression in goat skeletal muscle satellite cells (SMSCs). Immunocytochemistry analysis of the goat adiponectin protein using a human anti-adiponectin antibody in SMSCs (A) and compared with controls (B). Arrows indicate positive staining.
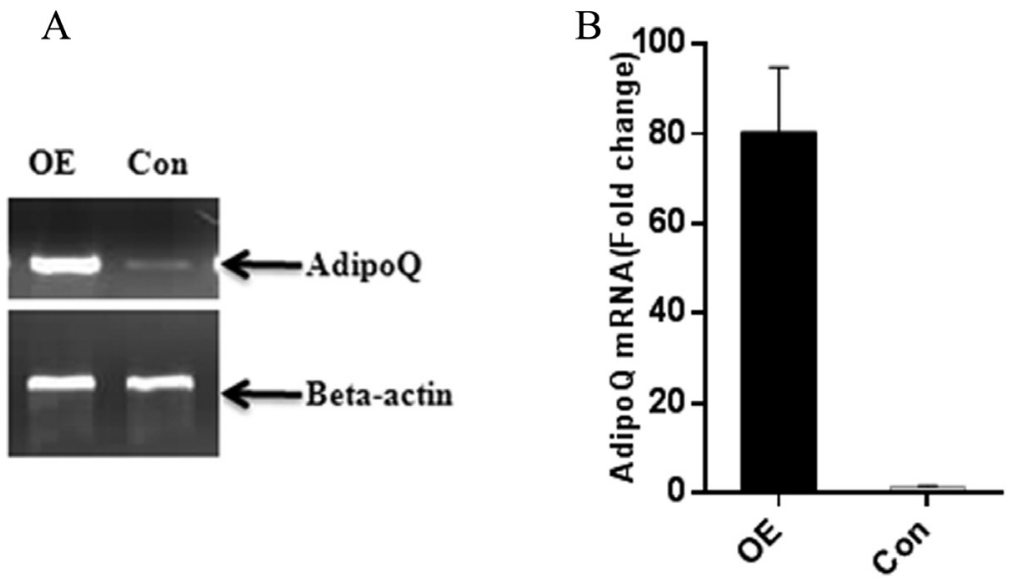

Figure 6. The effect of goat adiponectin overexpression. Cells were transfected with the pEGFP-N1-AD (OE) plasmid and then harvested for mRNA extraction after 48 hours. Effective overexpression of goat adiponectin was identified by semi-quantitative reverse transcription-polymerase chain reaction (RT-PCR) (A) and quantitative PCR (B). Cells transfected with the pEGFP-N1 plasmid were used as the control (Con).

and studies have proven that the C1Q domain has particular functions, including inducing SMC differentiation and fusion [24] and enhancing muscle fat oxidation and glucose transport [12]. The two receptors of adiponectin, AdipoR1 and AdipoR2, are seven-transmembrane domain proteins, but their protein structures lack homology with other mammalian proteins. In general, AdipoR1 and AdipoR2 are activated by different types of adiponectin. However, Narasimhan found that in yeast, the AdipoR1 and AdipoR2 proteins interact with osmotin, which has no sequence homology but does possess structural similarity to adiponectin [25]. This finding indicates that AdipoR1 and AdipoR2 might serve as receptors for other proteins.

Previous studies have shown that chicken AdipoQ is predominantly expressed in adipose tissue, whereas AdipoR 1 , and AdipoR2 are widely expressed in various tissues. AdipoR1
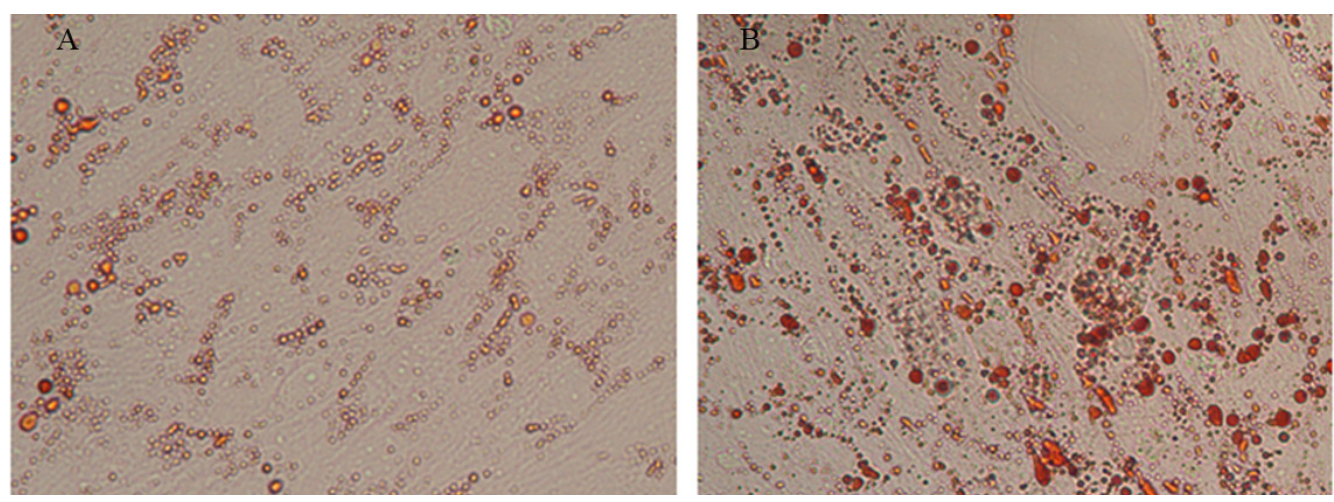

Figure 7. Lipid accumulation in cells of the AdipoQ-overexpression and control groups after $7 \mathrm{~d}$ of adipogenic differentiation, as determined by oil red 0 staining. 

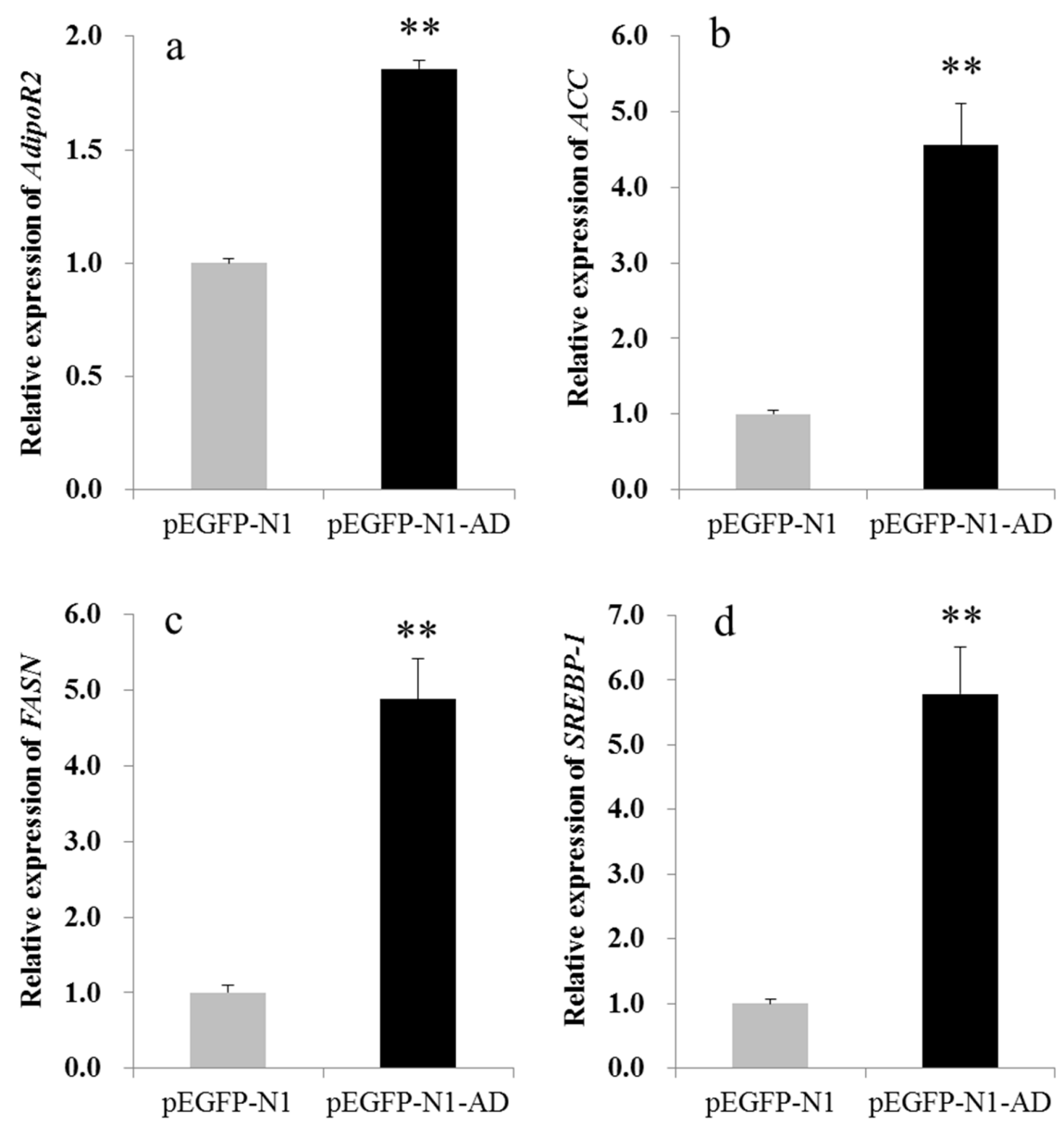

Figure 8. Expression levels of AdipoR2 (a), ACC (b), FASN (c) and SREBP-1 (d) in skeletal muscle satellite cells (SMSCs) overexpressing adiponectin. The expression levels of AdipoR2, ACC, FASN and SREBP-1 in adiponectin-overexpressing cells were 1.85, 4.55, 4.88, and 4.25 times, respectively, higher than in the control group. The results were normalized to $\beta$-actin expression, and expression in the control group was set as 1. Error bars represent $S E(n=3)$, ** $p<0.01$. AdpoR, adiponectin receptor; $A C C$, acetyl-CoA carboxylase; FASN, fatty-acid synthase; SREBP-1, sterol regulatory element-binding protein-1.

mRNA is highly expressed in skeletal muscles, adipose tissue and the diencephalon; AdipoR2 mRNA is most highly expressed in adipose tissue, followed by skeletal muscles and the liver [26]. Research in pigs showed that AdipoQ mRNA expression is abundant in adipose tissue, that AdipoR1 mRNA expression is abundant in skeletal muscle and adipose tissues, and that AdipoR $2 \mathrm{mRNA}$ is predominantly expressed in adipose and skeletal muscle tissues [27]. Overall, these three genes are mainly expressed in adipose and skeletal muscle tissues, where they are responsible for energy metabolism. Hence, the observed tissue distribution profile corresponds with their function in regulating lipid and fatty acid metabolism. In our study, the mRNA tissue distribution of AdipoQ and its two receptor genes was similar to that in chickens and pigs, suggesting a similar function among species.

A previous report stated that adiponectin expression and secretion are increased during the human adipocyte differentiation process, which is regarded as a distinctive marker of adipocyte differentiation [28]. Overexpression of adipo- nectin in 3T3-L1 fibroblasts increases the number and size of lipid droplets and promotes adipogenic differentiation [14]. Accordingly, these results suggest that adiponectin is able to promote fatty acid synthesis and adipocyte differentiation.

In this study, the adiponectin fusion protein was only found in the cytoplasm of SMSCs overexpressing the AdipoQ gene, indicating that adiponectin functions in the cytoplasm. Based on our qPCR results, the expression levels of AdipoR2, ACC, $F A S N$, and SREBP-1 were significantly up-regulated, suggesting that adiponectin interacts with AdipoR2 to regulate expression of ACC, FASN, and SREBP-1 genes in SMSCs. It has been reported that ACC and FASN are two important factors for de novo fatty acid synthesis; ACC catalyzes the production of malonyl-CoA from acetyl-CoA, which is then converted into long-chain fatty acids by FASN [29]. Moreover, SREBP-1 is an important transcription factor that regulates ACC and FASN transcription for fat metabolism and adipogenic differentiation [30]. Thus, increased expression of these three adipogenic genes may result in fatty acid synthesis and 
lipid accumulation, which indicates that adiponectin may promote fatty acid synthesis and adipogenic differentiation.

\section{CONFLICT OF INTEREST}

We certify that there is no conflict of interest with any financial organization regarding the material discussed in the manuscript.

\section{ACKNOWLEDGMENTS}

This work was supported by grants from the High Education Doctorial Subject Research Program (20115103120011), the Research and Development Project of Liangshan (16YYJS0111) and the Technology Support Program of Sichuan Province (2014NZ0001 \& 2015NZ0112).

\section{REFERENCES}

1. Scherer PE, Williams S, Fogliano M, Baldini G, Lodish HF. A novel serum protein similar to C1q, produced exclusively in adipocytes. J Biol Chem 1995;270:26746-9.

2. Katsiougiannis S, Kapsogeorgou EK, Manoussakis MN, Skopouli FN. Salivary gland epithelial cells: a new source of the immunoregulatory hormone adiponectin. Arthritis Rheum 2006;54:2295-9.

3. Staiger H, Kausch C, Guirguis A, et al. Induction of adiponectin gene expression in human myotubes by an adiponectincontaining HEK293 cell culture supernatant. Diabetologia 2003;46:956-60.

4. Berner HS, Lyngstadaas SP, Spahr A, et al. Adiponectin and its receptors are expressed in bone-forming cells. Bone 2004; 35:842-9.

5. Fruebis J, Tsao TS, Javorschi S, et al. Proteolytic cleavage product of $30-\mathrm{kDa}$ adipocyte complement-related protein increases fatty acid oxidation in muscle and causes weight loss in mice. Proc Natl Acad Sci USA 2001;98:2005-10.

6. Yamauchi T, Kamon J, Ito Y, et al. Cloning of adiponectin receptors that mediate antidiabetic metabolic effects. Nature 2003;423:762-9.

7. Yamauchi T, Nio Y, Maki T, et al. Targeted disruption of AdipoR1 and AdipoR2 causes abrogation of adiponectin binding and metabolic actions. Nat Med 2007;13:332-9.

8. Fang X, Fetros J, Dadson KE, Xu A, Sweeney G. Leptin prevents the metabolic effects of adiponectin in L6 myotubes. Diabetologia 2009;52:2190-200.

9. Yoon MJ, Lee GY, Chung JJ, et al. Adiponectin increases fatty acid oxidation in skeletal muscle cells by sequential activation of AMP-activated protein kinase, p38 mitogen-activated protein kinase, and peroxisome proliferator-activated receptor alpha. Diabetes 2006;55:2562-70.

10. Niu M, Xiang L, Liu Y, et al. Adiponectin induced AMPK impairment mediates insulin resistance in Bama mini-pig fed high-fat and high-sucrose diet. Asian-Australas J Anim Sci 2017;30:1190-7.

11. Shapiro L, Scherer PE. The crystal structure of a complement1q family protein suggests an evolutionary link to tumor necrosis factor. Curr Biol 1998;8:335-40.

12. Tomas E, Tsao TS, Saha AK, et al. Enhanced muscle fat oxidation and glucose transport by ACRP30 globular domain: acetyl-CoA carboxylase inhibition and AMP-activated protein kinase activation. Proc Natl Acad Sci USA 2002;99:16309-13.

13. Iwabu M, Yamauchi T, Okada-Iwabu M, et al. Adiponectin and AdipoR1 regulate PGC-1alpha and mitochondria by $\mathrm{Ca}(2+)$ and AMPK/SIRT1. Nature 2010;464:1313-9.

14.Fu Y, Luo N, Klein RL, Garvey WT. Adiponectin promotes adipocyte differentiation, insulin sensitivity, and lipid accumulation. J Lipid Res 2005;46:1369-79.

15. Meng X, Yang J, Cao Y, et al. Increasing fatty acid production in E. coli by simulating the lipid accumulation of oleaginous microorganisms. J Ind Microbiol Biotechnol 2011;38:919-25.

16. Chakravarty B, Gu Z, Chirala SS, Wakil SJ, Quiocho FA. Human fatty acid synthase: structure and substrate selectivity of the thioesterase domain. Proc Natl Acad Sci USA 2004;101:1556772 .

17. White UA, Stephens JM. Transcriptional factors that promote formation of white adipose tissue. Mol Cell Endocrinol 2010; 318:10-4.

18. Taniguchi M, Guan LL, Zhang B, et al. Adipogenesis of bovine perimuscular preadipocytes. Biochem Biophys Res Commun 2008;366:54-9.

19. Johnson PF. Molecular stop signs: regulation of cell-cycle arrest by C/EBP transcription factors. J Cell Sci 2005;118:2545-55.

20.Lefterova MI, Zhang Y, Steger DJ, et al. PPARgamma and C/ EBP factors orchestrate adipocyte biology via adjacent binding on a genome-wide scale. Genes Dev 2008;22:2941-52.

21. Ren J, Wen L, Gao X, et al. DOG 1.0: illustrator of protein domain structures. Cell Res 2009;19:271-3.

22. Robinson K, Kruger P, Phillips LK, Prins J, Venkatesh B. Effect of statin therapy on plasma adiponectin concentrations in patients with the sepsis syndrome: a preliminary investigation. Intensive Care Med 2011;37:1388-9.

23.Shams M, Rasekhi Kazerouni A, Ostovan MA, Omrani GR. The relationship between serum adiponectin levels with the presence and severity of coronary artery disease. Arch Iran Med 2012;15:611-6.

24. Fiaschi T, Cirelli D, Comito G, et al. Globular adiponectin induces differentiation and fusion of skeletal muscle cells. Cell Res 2009;19:584-97.

25.Narasimhan ML, Coca MA, Jin J, et al. Osmotin is a homolog of mammalian adiponectin and controls apoptosis in yeast through a homolog of mammalian adiponectin receptor. Mol Cell 2005;17:171-80.

26. Ramachandran R, Ocon-Grove OM, Metzger SL. Molecular 
cloning and tissue expression of chicken AdipoR1 and AdipoR2 complementary deoxyribonucleic acids. Domest Anim Endocrinol 2007;33:19-31.

27.Ding ST, Liu BH, Ko YH. Cloning and expression of porcine adiponectin and adiponectin receptor 1 and 2 genes in pigs. J Anim Sci 2004;82:3162-74.

28. Körner A, Wabitsch M, Seidel B, et al. Adiponectin expression in humans is dependent on differentiation of adipocytes and down-regulated by humoral serum components of high molecular weight. Biochem Biophys Res Commun 2005;337:
540-50.

29. Kim CW, Moon YA, Park SW, et al. Induced polymerization of mammalian acetyl-CoA carboxylase by MIG12 provides a tertiary level of regulation of fatty acid synthesis. Proc Natl Acad Sci USA 2010;107:9626-31.

30. Osborne TF, Espenshade PJ. Evolutionary conservation and adaptation in the mechanism that regulates SREBP action: what a long, strange tRIP it's been. Genes Dev 2009;23:257891. 